\title{
The rocky road to biomolecules
}

\begin{abstract}
A natural chemical reaction that occurs below the sea floor makes the amino acid tryptophan without biological input. This finding reveals a process that might have helped life on Earth to begin. SEE ARTICLE P.59
\end{abstract}

\section{JOHN A. BAROSS}

$\mathrm{R}$ obert Frost's poem Fire and Ice ponders which of these two will eventually cause life on Earth to cease. Conversely, scientists have long been interested in whether life on this planet originated in hot or cold conditions. Did life arise in a hot volcanic environment ${ }^{1,2}$ or, as Charles Darwin suggested in a letter (see go.nature.com/2q8w3n5), in "some warm little pond"? Might we need to invoke a global setting that includes ice ${ }^{3}$ or would an ocean-floor setting suffice ${ }^{1-5}$ ? On page 59, Ménez et al. ${ }^{6}$ report an analysis of rocky material below the ocean floor at sites called serpentinizing hydrothermal vents, which are hot springs that discharge alkaline, gasrich water. The authors provide evidence that a chemical reaction occurs there that could have set the stage for life to begin - the generation of an amino acid by a process that is not biologically mediated.

A well-characterized form of hydrothermal vent on the ocean floor, called black-smoker chimneys, occurs in a volcanic, magma-rich setting. These vents emit acidic fluids into the ocean that have high concentrations of gases and also of metals that are in a chemically reduced form.

However, in 2000, the serendipitous discovery of a mid-Atlantic ocean-floor site called Lost City revealed another type of hydrothermal-vent environment ${ }^{7}$. The Lost City vents were found to arise by a process termed serpentinization - a chemical interaction between water and a type of rock called peridotite that contains minerals enriched in magnesium, iron and silica. Serpentinization generates alkaline conditions that aid the formation of majestic carbonate-rich towers (Fig. 1). Serpentinization also produces hydrogen and a variety of organic molecules, including formate, acetate and pyruvate, which might be important in supporting the microbial life at Lost City and could also have been used in the biochemical steps that led to life on Earth ${ }^{8,9}$. Studies of Lost City have provided a treasure trove of scientific discoveries that have greatly altered our understanding of hydrothermal vents and the geological history of the early Earth ${ }^{10}$. They have also led to testable hypotheses about possible

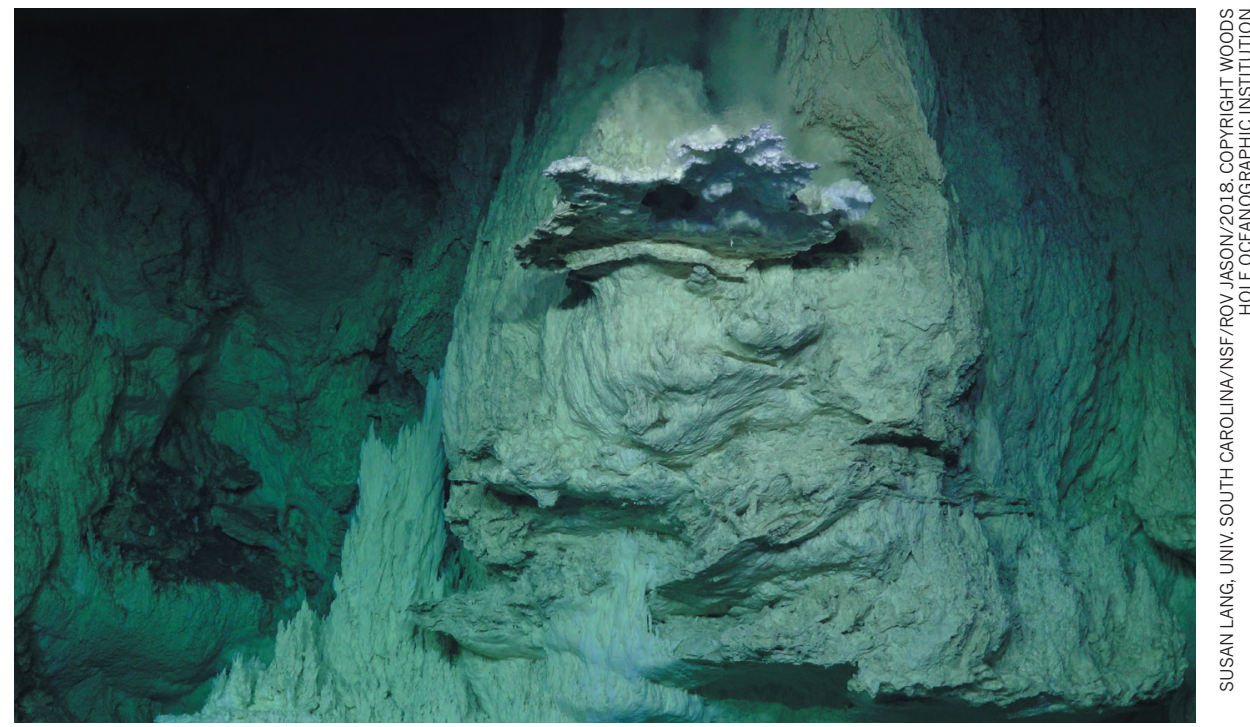

Figure 1 | Carbonate towers in hydrothermal vents at the Lost City site on the floor of the Atlantic Ocean.

environmental settings for the origin of life.

In laboratory experiments that simulate conditions in magma-hosted hydrothermal vents, amino acids can be synthesized by chemical reaction ${ }^{11}$ that do not require biological input. However, whether such abiotic generation of amino acids can occur in serpentinizing hydrothermal vents was unknown. Amino acids have been detected ${ }^{12}$ in fluids emanating from Lost City, but their source was undetermined.

To investigate further, Ménez and colleagues analysed material from rock samples retrieved by drilling around 170 metres below the ocean floor at Lost City. The authors present extensive evidence for the presence of the nitrogen-containing amino acid tryptophan in a context in which it was unlikely to have been produced by a biologically mediated process. They report data obtained from three high-resolution techniques that are consistent with the presence of tryptophan. The researchers also found other organic compounds that might be intermediates in the synthesis of tryptophan. Ménez et al. propose that this synthesis could be the result of Friedel-Crafts reactions because they found the molecule indole, which is an intermediate organic compound in the synthesis of tryptophan by this type of reaction. Their case for the abiotic synthesis of tryptophan is strengthened by the absence of other amino acids that would be present if a biological source was there, such as microbial contamination of the rock sample.

To extend Ménez and co-workers' report of the abiotic synthesis of tryptophan, future studies at Lost City should try to collect adequate volumes of fluid to determine a structural property, called chirality, of the tryptophan present. Molecules can exist in two mirror-image chiral forms. Synthesis of a molecule by a non-biological process generally results in equal proportions of these two forms, whereas biologically synthesized amino acids are usually made in predominantly one form or the other.

The authors' work also sheds light on the long-standing mystery of what mechanism reduces nitrogen molecules $\left(\mathrm{N}_{2}\right)$ to ammonia under hydrothermal-vent conditions. In most of the cases in which ammonia has been detected in hydrothermal-vent environments, it was found to originate from buried sediment sources of organic material and not from abiotic synthesis in the hydrothermal vents ${ }^{13}$. Ménez and colleagues propose that saponite, an iron-containing clay mineral that they detected, and which is reported ${ }^{14}$ to be a catalyst for the synthesis of organic compounds and the reduction of $\mathrm{N}_{2}$ to ammonia, might be involved in tryptophan synthesis. Abiotic generation of a source of ammonia, together 
with saponite's proposed catalysis of heterocyclic-amine molecules such as tryptophan, also raises the possibility of abiotic synthesis of other heterocyclic amines called pyrimidines and purines, which are components of the nucleic acids DNA and RNA. Moreover, saponite has the potential to promote the formation of organic polymers ${ }^{15,16}$.

Beyond the potential for the synthesis and accumulation of organic compounds that were probably important in the origin of life, serpentinization has two other characteristics that have intriguing implications regarding the origin of life and the establishment of habitable conditions ${ }^{5,8}$. One characteristic is that serpentinization produces heat. The gradient of temperature can reach more than $200^{\circ} \mathrm{C}$ at the site of the serpentinization reaction ${ }^{4}$. This, in turn, promotes hydration and therefore expansion of rocks, which is the other intriguing characteristic of serpentinization. However, if part of the ocean floor 'sinks' (subducts) into Earth's interior as tectonic plates move, the greater heat and pressure encountered on its descent into the deep subsurface region would reverse such serpentinization, and the water released during this reversal could help to give rise to volcanoes on the ocean floor ${ }^{14}$. This, in turn, might help to recycle key elements that support life.

The geological record at the time of life's origin, 3.5 billion to 4.4 billion years ago, is enriched in iron- and magnesium-containing minerals (characteristic of the rocks that form Earth's mafic crust), and in other elements that could have been extracted from rock in a process mediated by high-temperature water, strongly pointing to hydrothermal activity at that time. Yet, during the first billion years of Earth's history, the heat from the mantle was too great for plate tectonics to occur ${ }^{17,18}$. Consequently, heat would have been lost from Earth's interior mainly through volcanoes on the ocean floor. Earth's crust would have been rich in silicate minerals and iron ${ }^{17,18}$, allowing high rates of serpentinization and producing high concentrations of hydrogen and organic compounds.

Extensive circulation of seawater through volcanic rock during this time might have resulted in heat, fluid and gas ascending from the depths to create convective cells - a phenomenon characterized by currents due to density differences in the liquids or gases present. In the volcanic-rock environment, this could have led to associated gradients of temperature, pressure, chemical composition and wet-dry cycles (hydration-dehydration cycles known to promote chemical reactions that include the polymerization of organic compounds). Regardless of how life originated and in what environmental setting it was first established, serpentinization probably had an important role in facilitating the availability of organic chemicals required for life.

Understanding serpentinization at Lost City has wider scientific implications. Saturn's icy moon Enceladus has many of the chemical properties known to support life ${ }^{19,20}$ that are seen in serpentinizing environments such as those of Lost City. Whether or not Enceladus, or indeed other icy moons such as Jupiter's Europa, could or did support life, they nevertheless could provide insight into geochemical processes that might lead to life. Such geochemical analysis seems to support the hypothesis that hydrothermal systems might have had an essential role in the origin of life. A more far-reaching implication of the work by Ménez and colleagues, and of others investigating hydrothermal vents, is that efforts to understand the characteristics of these settings might aid efforts to search for life beyond Earth. A planetary body with evidence of geophysical properties, including plate tectonics and hydrothermal systems, might have a higher probability of acquiring and supporting carbon-based life than planetary bodies lacking such geophysical properties. If true, then targeting such planets might also increase our probability of finding such life. $\square$

John A. Baross is in the School of Oceanography and the Astrobiology Program, University of Washington, Seattle, Washington 98195, USA. e-mail:jbaross@u.washington.edu

1. Baross, J. A. \& Hoffman, S. E. Orig. Life Evol. Biosph. 15, 327-345 (1985).

2. Martin, W. \& Russell, M. J. Phil. Trans. R. Soc. Lond. B 362, 1887-1925 (2007).

3. Stüeken, E. E. et al. Geobiology 11, 101-136 (2013).

4. Martin, W., Baross, J., Kelley, D. \& Russell, M. J. Nature Rev. Microbiol. 6, 805-814 (2008).

5. Preiner, M. et al. Life 8, 41 (2018).

6. Ménez, B. et al. Nature 564, 59-63 (2018).

7. Kelley, D. et al. Nature 412, 145-149 (2001).

8. Lang, S. Q., Butterfield, D. A., Schulte, M., Kelley, D. S. \& Lilley, M. D. Geochim. Cosmochim. Acta 74, 941-952 (2010).

9. Schrenk, M. O., Brazelton, W. J. \& Lang, S. Q. Rev. Miner. Geochem. 75, 575-606 (2013).

10.Sleep, N. H., Bird, D. K. \& Pope, E. C. Phil. Trans. R. Soc. B 366, 2857-2869 (2011).

11.Hennet, R. J., Holm, N. G. \& Engel, M. H. Naturwissenschaften 79, 361-365 (1992).

12.Lang, S. Q., Früh-Green, G. L., Berncisconi, S. M. \& Butterfield, D. A. Geobiology 11, 154-169 (2013).

13.Lilley, M. D. et al. Nature 364, 45-47 (1993).

14.Kooli, F. \& Jones, W. Clay Miner. 32, 633-643 (1997).

15. Ferris, J. P., Hill, A. R. Jr, Liu, R. \& Orgel, L. E. Nature 381, 59-61 (1996).

16. Hazen, R. M. \& Sverjensky, D. A. Cold Spring Harb. Perspect. Biol. 2, a002162 (2010).

17.Dhuime, B., Wuestefeld, A. \& Hawkesworth, C. J. Nature Geosci. 8, 552-555 (2015).

18. Tang, M., Chen, K. \& Rudnick, R. L. Science 351 , 372-375 (2016).

19.Waite, J. H. et al. Science 311, 1419-1422 (2006).

20.Waite, J. H. et al. Science 356, 155-159 (2017).

This article was published online on 7 November 2018.

\section{CONDENSED-MATTER PHYSICS}

\section{Elusive spin textures discovered}

\section{Magnetic materials can host a range of structures called spin textures. Two such textures - a meron and an antimeron - have been observed experimentally for the first time, in a material known as a chiral magnet. SEE LETTER P.95}

\section{SEONGHOON WOO}

$\mathrm{M}$ agnetic moments (spins) in magnetic materials can form various structures known as spin textures. In most cases, spins of neighbouring atoms tend to align parallel or antiparallel to each other, resulting in ferromagnets or antiferromagnets, respectively. However, in some materials called chiral magnets that have unusual physical interactions between spins owing to a peculiar crystalline or multilayer structure, spins align in an intricate fashion: a topological spin texture. On page 95, Yu et al. ${ }^{1}$ report the first experimental evidence for two such textures, and observe transitions between textures that could have applications in spinbased electronics (spintronics).

The archetypal topological spin texture is a small, swirling magnetic knot known as a magnetic skyrmion (Fig. 1). In a skyrmion, the orientation of spins rotates progressively from the up direction at the edge of the texture to the down direction at the centre, or vice versa. The properties of a skyrmion can be characterized by a value of either -1 or +1 for a quantity called the topological charge.

Magnetic skyrmions were discovered ${ }^{2}$ in 2009 and were observed at room temperature $^{3}$ in 2015. Since then, they have been at the centre of research in many scientific and technical fields, for at least three reasons ${ }^{4}$. First, they can be very stable, owing to a phenomenon called topological protection. Second, they can be extremely tiny (with diameters in the nanometre range), which means that they could be used in future nanotechnology. And third, they exhibit energy-efficient current-driven behaviour that is suitable for next-generation low-energy spintronic devices such as those involving computer memory, logic, information transmission and 